\title{
Hormônio de crescimento em crianças e adolescentes com fibrose cística
}

\author{
Growth hormone in children and adolescents with cystic fibrosis
}

Pollyana Garcia Amorim', Thaís de Barros Mendes', Lílian Santiago

Pinho de Oliveira², Gil Guerra-Júnior ${ }^{3}$, José Dirceu Ribeiro ${ }^{4}$

\section{SUMÁRIO}

O hormônio de crescimento recombinante humano ( $\mathrm{rhGH}$ ) pode melhorar o ganho ponderal, o crescimento físico e as condições clínicas e pulmonares na fibrose cística (FC). Entretanto, o uso rotineiro, embora promissor, não está estabelecido na literatura. O objetivo deste trabaIho foi verificar os benefícios do rhGH em crianças e adolescentes com FC. Realizou-se uma revisão sistemática nas bases de dados PubMed, Lilacs, SciELO, Cochrane, no período de 20002010, utilizando-se as palavras-chave: "fibrose cística", “hormônio de crescimento", "crianças" e "adolescentes". Foram encontrados 77 artigos, sendo incluídos 11 estudos randomizados controlados, com 290 crianças e adolescentes com FC. 0 uso em curto prazo (1-24 meses) do rhGH melhorou estatura, peso, velocidade de crescimento, massa óssea e componentes da função pulmonar. Efeitos adversos como diabetes não foram observados nos estudos. $\mathrm{O}$ uso em curto prazo do rhGH melhorou o crescimento e a composição corporal em pacientes com FC. Arq Bras Endocrinol Metab. 2011;55(9):671-6

Descritores

Hormônio do crescimento; fibrose cística; saúde do adolescente e da criança

\section{SUMMARY}

The recombinant human growth hormone ( $\mathrm{rhGH}$ ) can improve weight gain, physical growth, clinical and lung in cystic fibrosis (CF). However, the routine use, although promising, is not established in the literature. The objective of this study was to assess the benefits of rhGH in children and adolescents with CF. We conducted a systematic review in the database PubMed, Lilacs, SciELO and Cochrane, in the period 2000-2010, using the keywords: "cystic fibrosis", "growth hormone", "children" and "adolescents". We found 77 articles and included 11 randomized controlled trials, with 290 children and adolescents with CF. The short-term use (1-24 months) of rhGH improved the height, weight, growth rate, bone mineral content and components of pulmonary function. Adverse effects, like diabetes, were not observed in the studies. The short-term use of rhGH improved growth and body composition in patients with CF. Arq Bras Endocrinol Metab. 2011;55(9):671-6

\section{Keywords}

Growth hormone; cystic fibrosis; adolescent health and children

\section{INTRODUÇÃO}

$\mathrm{A}^{\mathrm{n}}$ fibrose cística (FC) é a doença genética com herança autossômica recessiva mais comum na população de origem caucasiana, afetando cerca de 1 a 2.500 nascidos vivos. É causada por defeitos no gene CFTR (Cystic Fibrosis Transmembrane conductance Regulator)
Faculdade de Ciências Médicas (FCM), Universidade Estadual de Campinas (Unicamp),

Campinas, SP, Brasil

2 Escola Baiana de Medicina e Saúde Pública (EBMSP),

Salvador, BA, Brasil

${ }^{3}$ Departamento de Pediatria Endocrinologia Pediátrica, FCM, Unicamp, Campinas, SP, Brasil ${ }^{4}$ Departamento de Pediatria Pneumologia Pediátrica, FCM, Unicamp, Campinas, SP, Brasil
Correspondência para: Pollyana Garcia Amorim Rua Joaquim José Albino Pereira, 102 13040-480 - Campinas, SP, Brasil pollyanag@gmail.com

Recebido em 15/Abr/2011 Aceito em 28/Out/2011 localizado em 7q31.2, que codifica uma proteína localizada na membrana apical das células epiteliais das vias aeríferas, do pâncreas, das glândulas salivares e sudoríparas, do intestino e do aparelho reprodutor, cuja função é regular o canal de cloro. Mais de 1.800 mutações foram associadas à FC, sendo a F508del a mais frequente (1). 
Em indivíduos homozigotos afetados, a doença em geral tem apresentação sistêmica, com predomínio do quadro clínico da doença pulmonar crônica e da insuficiência pancreática exócrina (2). Avanços no tratamento médico, incluindo nutrição oral agressiva (ingestão calórica acima de $120 \%$ das taxas diárias recomendadas), suplementação adequada de enzima pancreática e vitaminas, fisioterapia e tratamento antimicrobiano, têm melhorado a sintomatologia e o prognóstico da doença, com vários pacientes atingindo a vida adulta $(2,3)$. Atualmente, na maioria dos centros, cerca de metade dos pacientes sobrevive à terceira década de vida $(4,5)$. Entretanto, a altura e o peso dos pacientes com FC geralmente se apresentam nos percentis mais baixos, e o baixo peso está relacionado ao aumento da morbimortalidade (6).

A FC é uma doença inflamatória crônica com altas concentrações séricas e teciduais de numerosas interleucinas pró-inflamatórias, principalmente IL-1, IL-2 e IL-8, que, por si, podem aumentar o gasto energético e causar anorexia, resultando em alterações ponderais e estaturais. O processo inflamatório crônico tanto afeta a produção e a secreção do hormônio de crescimento (GH) como inibe o crescimento em nível tecidual $(7,8)$. Dessa maneira, na FC, a suplementação nutricional isolada pode ser insuficiente para ocasionar a recuperação adequada do peso e da estatura.

Muitos pacientes com FC são desnutridos (27,5\% das mulheres e $53,5 \%$ dos homens, entre 18 e 20 anos de idade) e, também, apresentam baixa estatura (3\% das mulheres e $13 \%$ dos homens, entre 18 e 20 anos de idade). Pacientes com FC apresentam altura abaixo do alvo parental e a altura adulta em geral é reduzida, atingindo em média os percentis 25 a 45 para homens e mulheres (9). Em um estudo com 17.857 pacientes com FC, a média da altura foi o percentil 21 e a do peso foi o percentil 9 (10). A deficiência de ganho no peso e na estatura e o atraso na maturação óssea já ocorrem nos primeiros anos de vida e depois progridem lentamente ou se mantêm estáveis durante a infância, para se agravarem novamente na época próxima à puberdade, quando as alterações no crescimento e na maturação ficam muito evidentes, à semelhança de quadros mais graves de retardo constitucional do crescimento e desenvolvimento (10-14).

As deficiências ponderal e estatural são clinicamente relevantes não somente porque afetam a qualidade de vida $(3,15)$, mas também porque a gravidade delas pode estar mais relacionada à gravidade da doença pul- monar que à deficiência pancreática e à morbimortalidade em geral em pacientes com FC $(6,10)$.

$\mathrm{O}$ eixo $\mathrm{GH} /$ fator de crescimento semelhante à insulina (IGF) em pacientes com FC mostra valores médios elevados de GH e baixos de IGFl, denotando algum grau de resistência adquirida ao GH (16-18). Porém, são poucos os artigos na literatura que mostram o efeito do $\mathrm{GH}$ recombinante humano ( $\mathrm{rhGH}$ ) no tratamento do distúrbio de crescimento de pacientes com FC.

Embora o ganho de estatura seja o melhor efeito conhecido do rhGH, ele também contribui com outras funções metabólicas como aumentar a retenção de cálcio e a mineralização óssea; aumentar a massa muscular; induzir a síntese proteica e o crescimento de órgãos e tecidos. O rhGH também estimula o sistema imunológico e tem um papel na homeostase de energia do organismo, reduzindo o consumo de glicose pelo fígado, efeito oposto ao da insulina; contribui para a manutenção e o funcionamento das ilhotas pancreáticas; promove lipólise, que resulta na redução do tecido adiposo corporal e no aumento de ácidos graxos livres e glicerol na corrente sanguínea $(9)$.

Nesses estudos realizados com rhGH em pacientes com FC, a sua utilização tem sido proposta com o intuito de aumentar a mineralização óssea, melhorar a síntese e diminuir a degradação de proteínas, e com isso melhorar o crescimento em geral $(9,18,19)$, porém com o cuidado para não precipitar ou agravar a disfunção pancreática de células beta, com hiperglicemia, resistência insulínica e diabetes mellitus $(9,20)$.

No entanto, ainda são muito poucos os estudos com rhGH em pacientes com FC e, também, são poucos os centros de referência no manejo desses pacientes que utilizam esse tipo de tratamento $(9,19,20)$. Portanto, o objetivo deste estudo foi realizar uma revisão sistemática da literatura para verificar os benefícios do uso de rhGH em crianças e adolescentes com FC.

\section{MATERIAIS E MÉTODOS}

Realizou-se uma revisão sistemática, incluindo artigos publicados em língua portuguesa, inglesa, francesa e espanhola, no período de 2000 a 2010. A busca de referências se fez por meio da exploração de bancos de dados das seguintes bases: PubMed-Medline, Lilacs, SciELO, Cochrane, utilizando os termos: "cystic fibrosis", "growth hormone", "children", "adolescent" e suas traduções correspondentes, em combinações variadas. 
Além disso, as revisões sobre o tema e as listas de referências de todos os artigos incluídos foram consultadas, em busca de novos artigos para inclusão.

Após essa etapa, foi iniciado o processo de seleção dos estudos pela análise de títulos e resumos. O primeiro critério de inclusão utilizado foi a identificação de trabalhos potencialmente relevantes, considerando aqueles em que a amostra era constituída por crianças e/ou adolescentes com diagnóstico de FC e que foram publicados a partir de 2000. Nessa ocasião, foram excluídos os estudos com adultos e aqueles cujo objetivo era apenas descrever a doença.

\section{SÍNTESE DOS DADOS}

Foram encontrados, com as referidas palavras-chave, 43 artigos na primeira busca nas bases PubMed-Medline, 21 no site Central Cochrane e 13 no site SciELO-Lilacs.

Por meio da avaliação dos resumos incluídos nessa fase, foram definidos como critérios para recuperação de artigos completos: estudos de coorte, metanálises, revi- sões sistemáticas e ensaios clínicos randomizados, cujos resultados mostravam o uso do rhGH em crianças e/ou adolescentes com diagnóstico de FC. A revisão foi finalizada com a leitura dos artigos completos, e foram inclú́dos no texto final 11 estudos, que descreveram os resultados do uso do rhGH, sendo esses dispostos na tabela 1 .

Dos 11 artigos selecionados (total de 290 crianças e/ou adolescentes), 9 ensaios compararam o uso do rhGH com um grupo controle sem tratamento (2129), I utilizou um grupo controle com placebo (30) e 1 avaliou o uso do rhGH isolado, associado com glutamina e uso da glutamina isolada (31). A duração do tratamento variou de $6(24,30)$ a 12 meses $(21$ $23,25,26,28,29)$, entretanto em 1 a duração foi de apenas 1 mês (31) e em outro, de 24 meses (27).

A idade cronológica dos pacientes variou de 7 a 23 anos, sendo que em 7 estudos foram realizados apenas com crianças pré-púberes $(21-24,27,28,31), 1$ apenas com púberes $(26)$ e 3 com pré-púberes e púberes $(25,29,30)$. A dose variou de 0,23 a $0,49 \mathrm{mg} / \mathrm{kg}$ por semana, sendo a mais utilizada a de $0,3 \mathrm{mg} / \mathrm{kg}$ por semana.

Tabela 1. Visão geral dos estudos randomizados e controlados com uso de rhGH em pacientes com FC publicados entre 2000 e 2010

\begin{tabular}{|c|c|c|c|}
\hline Estudos (referência) & Amostra & rhGH & Principais variáveis avaliadas \\
\hline Hardin e cols., 2001 (22) & $\begin{array}{c}19 \text { pré-púberes } \\
10 \text { com e } 9 \text { sem rhGH }\end{array}$ & $\begin{array}{l}0,3 \mathrm{mg} / \mathrm{kg} / \mathrm{sem} \\
12 \mathrm{meses}\end{array}$ & $\begin{array}{c}\text { Leucina (aparecimento, oxidação e desaparecimento da forma não oxidativa)* } \\
\qquad \text { IGF1*, insulina*, TNF- } \alpha^{*}, \text { TG }\end{array}$ \\
\hline Hardin e cols., 2001 (23) & $\begin{array}{l}19 \text { pré-púberes } \\
10 \text { com e } 9 \text { sem rhGH }\end{array}$ & $\begin{array}{l}0,3 \mathrm{mg} / \mathrm{kg} / \mathrm{sem} \\
12 \mathrm{meses}\end{array}$ & Peso*, estatura* ${ }^{\star} C^{\star}, M^{*}{ }^{\star}$, função pulmonar ${ }^{\star}, A B^{\star}$, hospitalizações* ${ }^{\star}, T G$ \\
\hline Hutler e cols., 2002 (24) & $\begin{array}{l}10 \text { pré-púberes } \\
6 \text { com e } 4 \text { sem rhGH }\end{array}$ & $\begin{array}{l}0,33 \mathrm{mg} / \mathrm{kg} / \mathrm{sem} \\
6 \text { meses }\end{array}$ & Peso*, estatura*, MM, tolerância ao exercício*, função pulmonar \\
\hline Schibler e cols., 2003 (25) & $\begin{array}{c}19 \text { (10-23 anos) } \\
10 \text { com e } 9 \text { sem rhGH }\end{array}$ & $\begin{array}{l}0,33 \mathrm{mg} / \mathrm{kg} / \mathrm{sem} \\
12 \mathrm{meses}\end{array}$ & Peso, $M^{*}{ }^{\star}$, tolerância ao exercício*, função pulmonar, $T G$ \\
\hline Darmaun e cols., 2004 (31) & $\begin{array}{l}9 \text { pré-púberes } \\
\text { rhGH } \pm \text { glutamina }\end{array}$ & $\begin{array}{l}0,3 \mathrm{mg} / \mathrm{kg} / \mathrm{sem} \\
1 \mathrm{mês}\end{array}$ & $\begin{array}{l}\text { rhGH isolado ou associado à glutamina reduziu a oxidação de leucina e } \\
\text { aumentou a síntese proteica }\end{array}$ \\
\hline Hardin e cols., 2005 (26) & $\begin{array}{l}25 \text { púberes } \\
13 \text { com e } 12 \text { sem rhGH }\end{array}$ & $\begin{array}{l}0,3 \mathrm{mg} / \mathrm{kg} / \mathrm{sem} \\
12 \mathrm{meses}\end{array}$ & $\begin{array}{l}\text { Peso*}^{\star} \text {, estatura* }{ }^{*} C^{\star}, I M C^{*}, M^{*}, C M 0^{*}, I G F 1^{\star} \text {, esteroides sexuais, } \\
\text { vitamina } D \text {, ingestão calórica, função pulmonar*, hospitalizações }{ }^{*}, T G\end{array}$ \\
\hline Hardin e cols., 2005 (27) & $\begin{array}{l}18 \text { pré-púberes } \\
9 \text { com e } 9 \text { sem rhGH }\end{array}$ & $\begin{array}{l}0,3 \mathrm{mg} / \mathrm{kg} / \mathrm{sem} \\
24 \mathrm{meses}\end{array}$ & 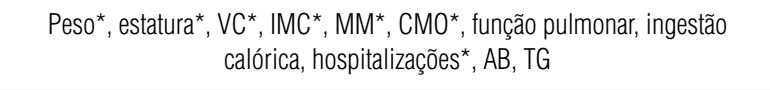 \\
\hline Hardin e cols., 2005 (28) & $\begin{array}{l}32 \text { pré-púberes } \\
16 \text { com e } 16 \text { sem rhGH }\end{array}$ & $\begin{array}{l}0,3 \mathrm{mg} / \mathrm{kg} / \mathrm{sem} \\
12 \mathrm{meses}\end{array}$ & $\begin{array}{l}\text { Peso*, estatura*, } V C^{\star}, I M C^{\star}, M M^{\star}, C M 0^{*} \text {, função pulmonar, ingestão } \\
\text { calórica, hospitalizações* }{ }^{*}, T G\end{array}$ \\
\hline Hardin e cols., 2006 (21) & $\begin{array}{c}61 \text { pré-púberes } \\
32 \text { com e } 29 \text { sem rhGH }\end{array}$ & $\begin{array}{c}0,3 \mathrm{mg} / \mathrm{kg} / \mathrm{sem} \\
12 \text { meses }\end{array}$ & Peso $^{\star}$, estatura* ${ }^{\star} \mathrm{VC}^{\star}, \mathrm{MM}^{\star}, \mathrm{CMO}^{\star}$, IGF1$^{\star}$, esteroides sexuais, TG \\
\hline Schnabel e cols., 2007 (30) & $\begin{array}{c}63 \text { (9-20 anos) } \\
42 \text { com e } 21 \text { sem rhGH }\end{array}$ & $\begin{array}{c}0,27 \text { ou } 0,49 \mathrm{mg} / \mathrm{kg} / \mathrm{sem} \\
6 \text { meses }\end{array}$ & Peso, estatura* ${ }^{\star} C^{\star}$, MM, IMC, IGF1*, IGFBP3*, função pulmonar, TG \\
\hline Grasemann e cols., 2008 (29) & $\begin{array}{c}15 \text { (12-18 anos) } \\
11 \text { com e } 4 \text { sem rhGH }\end{array}$ & $\begin{array}{c}0,27 \text { ou } 0,49 \mathrm{mg} / \mathrm{kg} / \mathrm{sem} \\
12 \mathrm{meses}\end{array}$ & NO soro e urina* ${ }^{*}$ AA no soro* \\
\hline
\end{tabular}

AA: aminoácidos; AB: uso de antibiótico; CMO: conteúdo mineral ósseo; IGF1: fator de crescimento semelhante à insulina tipo 1; IGFBP3: proteína ligadora 3 ao fator de crescimento semelhante à insulina; IMC: índice de massa corporal; MM: massa magra; NO: óxido nítrico; rhGH: hormônio de crescimento recombinante humano; TG: tolerância à glicose; TNF- $\alpha$ : fator de necrose tumoral; VC: velocidade de crescimento; *: $p<0,05$. 
A estatura foi avaliada em 7 estudos $(21,23,24,26-$ $28,30)$, em todos com significância estatística com a utilização do rhGH. A velocidade de crescimento foi avaliada em 6 estudos (21,23,26-28,30), em todos com significância estatística com a utilização do rhGH. O peso foi avaliado em 8 estudos $(21,23-28,30)$, em 6 (21,23,24,26-28) com significância estatística com a utilização do rhGH. O índice de massa corporal foi avaliado em 4 estudos (26-28,30), em 3 (26-28) com significância estatística com a utilização do rhGH. A massa magra foi avaliada em 8 estudos $(21,23-28,30)$, em todos, exceto l (30), com significância estatística com a utilização do rhGH. O conteúdo mineral ósseo foi avaliado em apenas 3 estudos $(21,26,27)$, em todos com significância estatística com a utilização do rhGH. As concentrações de IGFl foram avaliadas em 4 estudos $(21,22,26,30)$, em todos com significância estatística com a utilização do rhGH.

Sete estudos $(23-28,30)$ também avaliaram a função pulmonar, e na maioria dos estudos ocorreu melhora com o uso do rhGH, contudo apenas $2(23,26)$ demonstraram significância estatística. O uso de antibióticos foi avaliado em 3 estudos $(23,27,28)$, com diminuição significativa no seu uso no grupo com rhGH demonstrada apenas no estudo de Hardin e cols. (23). O número de hospitalizações foi avaliado em 4 estudos (23,26-28), e em todos foi observada diminuição desse número no grupo que usou rhGH. Apenas 1 estudo (29) teve como objetivo avaliar o efeito do rhGH na formação do óxido nítrico $(\mathrm{NO})$ sistêmico e nas vias aéreas, verificando-se que a concentração de L-arginina (substrato da síntese de $\mathrm{NO}$ ) aumentou no plasma e diminuiu no escarro.

Em relação aos efeitos hiperglicemiantes do rhGH, dos 11 estudos incluídos, apenas $3(24,29,31)$ não avaliaram a glicemia e a tolerância à glicose durante 0 estudo. Os demais 8 estudos (21-23,25-28,30) não encontraram modificação da tolerância à glicose quando compararam o grupo que utilizou rhGH com o grupo controle.

\section{DISCUSSÃO}

O desenvolvimento de pesquisas para o manejo de pacientes com FC tem contribuído intensamente para o avanço no conhecimento neste campo de atuação multidisciplinar. A presente revisão sistemática evidencia que o uso em curto prazo do rhGH foi benéfico para a melhora da estatura, do peso, da massa magra, do conteúdo mineral ósseo e de alguns componentes da função pulmonar em pacientes com FC, sem comprometimento da tolerância à glicose.

Nesta análise sistemática de 11 estudos publicados entre 2000 e 2010 foi observada melhora da estatura $(21,23,24,26-28,30)$ e da velocidade de crescimento $(21,23,26-28,30)$ com 6 a 24 meses de tratamento com rhGH em pacientes com FC. A dose média foi de $0,3 \mathrm{mg} / \mathrm{kg} / \mathrm{semana}$, que corresponde a uma dose maior que a dose de reposição fisiológica em pacientes com deficiência de GH (21-31), o que é justificado tendo em vista a existência de algum grau de resistência adquirida ao $\mathrm{GH}$ em pacientes com FC (16-18). Além da boa resposta clínica nesse curto período de tratamento, alguns estudos mostraram também que essa dose de rhGH foi suficiente para aumentar significativamente as concentrações séricas de IGFl $(21,22,26,30)$, ultrapassando essa resistência ao GH e permitindo uma resposta adequada de crescimento.

Também nessa análise sistemática de 11 estudos publicados entre 2000 e 2010 , foi observada melhora do peso $(21,23,24,26-28)$, do IMC (26-28) e da massa magra $(21,23-28)$ com 6 a 24 meses de tratamento com rhGH em pacientes com FC. Entre as explicações para a melhora ponderal e da composição corporal nesses pacientes com FC, está a ação anabolizante do rhGH, que permitiu melhora nutricional, com aumento do apetite, diminuindo o catabolismo proteico e permitindo a substituição de massa gorda por massa magra. Esse efeito pode ser evidenciado laboratorialmente nos estudos de Darmaun e cols. (31), que avaliaram os efeitos do rhGH associado ou não à glutamina no metabolismo de proteínas de pacientes com FC em uso de rhGH por quatro semanas, e no estudo de Hardin e cols. (22), que avaliaram o catabolismo de proteínas durante o uso de rhGH por 12 meses em pacientes com FC.

Ainda, nesta análise sistemática de 11 estudos publicados entre 2000 e 2010 , foi observada melhora do conteúdo mineral ósseo $(21,26,27)$. A maioria dos pacientes com FC pode apresentar baixa massa óssea devida à desnutrição, à deficiência de vitamina $\mathrm{D}$ e também à menor massa magra, que se correlaciona diretamente com a massa óssea. Além disso, o uso de corticosteroide e o hipogonadismo hipogonadotrófico funcional podem agravar a perda de massa óssea. O uso de rhGH em curto prazo nesse grupo de pacientes melhorou o conteúdo mineral ósseo, por melhorar o padrão nutricional, aumentar 
a massa magra e, também, por um possível efeito direto no osso, aumentando a atividade dos osteoblastos $(32,33)$.

Embora alguns trabalhos avaliados nesta análise sistemática de 11 estudos publicados entre 2000 e 2010 não tenham sido capazes de demonstrar efeitos benéficos do rhGH sobre a inflamação ou reparação pulmonar em pacientes com FC, outros mostraram melhora da capacidade vital e da ventilação máxima $(23-28,30)$, que pode ter ocorrido devido à associação positiva entre o crescimento físico longitudinal e o aumento do volume pulmonar $(25,26)$.

Em um estudo recente de metanálise, para avaliar os efeitos do rhGH em pacientes com FC, foram incluídos 10 ensaios clínicos controlados num total de 312 pacientes e 8 estudos observacionais com 58 pacientes, todos com uso de rhGH em curto prazo (máximo de 36 meses). Os autores concluíram que a utilização do rhGH melhorou o peso, a estatura, a massa óssea e quase todas as medidas de função pulmonar, além de diminuir o número de internações hospitalares. No entanto, com a exceção das internações, os benefícios nos desfechos finais à saúde não podem ser diretamente determinados até este momento devido ao pouco tempo de uso do rhGH (34).

Os pacientes com FC podem apresentar deficiência relativa à insulina com intolerância à glicose, evoluindo até diabetes mellitus, como resultado do dano das células betapancreáticas (35). Portanto, o uso de rhGH, especialmente em doses não farmacológicas, nesse grupo de pacientes com FC, poderia aumentar o risco de predispor ou agravar um estado de intolerância à glicose. Nesta revisão de 11 estudos de uso de rhGH em pacientes com FC, publicados entre 2000 e 2010 , apenas $3(24,29,31)$ não avaliaram a glicemia e a tolerância à glicose durante o estudo, e os demais 8 (21-23,25-28,30) não encontraram modificação da tolerância à glicose quando compararam o grupo que utilizou rhGH com o grupo controle.

Já em relação ao decréscimo do $\mathrm{NO}$ pulmonar e sistêmico, foi verificado que este pode ocorrer (29). O NO é sintetizado a partir da arginina e do oxigênio pela enzima sintase do óxido nítrico (NOS), sendo um composto nocivo para bactérias e vasodilatador sistêmico. Não é completamente compreendida a razão pela qual os pacientes com FC apresentam baixa concentração de NO nas vias aéreas. Acredita-se que isso ocorre devido à deficiência de L-arginina, substrato da NOS, que também pode ter efeito protetor sobre o estresse oxidativo pulmonar e defesa antioxidante. Grasemann e cols. (29) realizaram um estudo com o objetivo de verificar os efeitos do rhGH utilizando a concentração de aminoácidos no plasma e na urina e também nas vias aéreas pela medida do NO. Observaram que houve aumento do NO plasmático e urinário, porém este se apresentou diminuído quando exalado e também houve diminuição da L-arginina no escarro. Os autores concluíram que essa diminuição se deu por causa de uma menor oferta de substrato para a NOS, podendo ocorrer devido à interação do rhGH na formação do NO e também ao processo inflamatório crônico em pacientes com FC. Porém, não se sabe exatamente como esse mecanismo acontece.

\section{CONCLUSÃO}

Portanto, nesta revisão sistemática de 11 estudos com uso em curto prazo de rhGH em pacientes, a maioria pré-púberes, com FC, foi observada melhora da estatura, do peso, da massa magra, do conteúdo mineral ósseo e de alguns componentes da função pulmonar, sem comprometimento da tolerância à glicose. No entanto, os efeitos na composição corporal, massa óssea e tolerância à glicose não podem ser extrapolados para resultados de aquisição final, pois os estudos foram realizados apenas durante um curto prazo de tempo. Estudos com maior número de pacientes, com maior duração e com controle da previsão de estatura final e da tolerância à glicose são necessários para avaliar o real efeito do rhGH no ganho estatural desses pacientes e no seu efeito na tolerância à glicose. No entanto, não se pode deixar de ressaltar o ganho metabólico e nutricional observado, o que refletiu positivamente na função pulmonar, na diminuição do número de internações, na menor utilização de antibióticos e, consequentemente, na melhor qualidade de vida desses pacientes com FC.

Agradecimentos: os autores agradecem aos professores da disciplina de Crescimento e Desenvolvimento do Curso de Pós-Graduação em Saúde da Criança e do Adolescente da FCM-Unicamp, Drs. Antônio de Azevedo Barros-Filho e Angélica Maria Bicudo Zeferino, pela valiosa contribuição na confecção do presente artigo.

Declaração: os autores declaram não haver conflitos de interessecientífico neste estudo.

\section{REFERÊNCIAS}

1. OMIM - Online Mendelian Inheritance in Man. Disponível em: $<$ http://www.ncbi.nlm.nih.gov/omim>. 
2. Mogayzel PJ Jr, Flume PA. Update in cystic fibrosis 2010. Am J Respir Crit Care Med. 2011;183:1620-4.

3. Royce FH, Carl JC. Health-related quality of life in cystic fibrosis. Curr Opin Pediatr. 2011;23:535-40.

4. Alvarez AE, Ribeiro AF, Hessel G, Bertuzzo CS, Ribeiro JD. Cystic fibrosis at a Brazilian center of excellence: clinical and laboratory characteristics of 104 patients and their association with genotype and disease severity. J Pediatr (Rio J). 2004;80:371-9.

5. Ribeiro JD, Ribeiro MAGO, Ribeiro AF. Controversies in cystic fibrosis - from pediatrician to specialist. J Pediatr (Rio J). 2002;78(Suppl 2):S171-86.

6. Sharma R, Florea VG, Bolger AP, Doehner W, Florea ND, Coats AJS, et al. Wasting as an independent predictor of mortality in patients with cystic fibrosis. Thorax. 2001;56:746-50.

7. Hardin DS. GH improves growth and clinical status in children with cystic fibrosis - a review of published studies. Eur J Endocrinol. 2004;151(Suppl 1):S81-5.

8. Street MA, Ziveri MA, Spaggiari C, Viani I, Volta C, Grzincich GL, et al. Inflammation is a modulator of the insulin-like growth factor (IGF)/IGF-binding protein system inducing reduced bioactivity of IGFs in cystic fibrosis. Eur J Endocrinol. 2006;154:47-52.

9. Schnabel D. Cystic fibrosis - growth hormone treatment. In: Ranke MB, Price DA, Reiter EO (eds). Growth hormone therapy in Pediatrics -20 years of KIGS. Basel: Karger; 2007. p. 296-303.

10. FitzSimmons SC. The changing epidemiology of cystic fibrosis. J Pediatr. 1993;122:1-9.

11. Landon C, Rosenfeld RG. Short stature and pubertal delay in male adolescents with cystic fibrosis. Am J Dis Child. 1984;138:388-91.

12. Reiter EO, Stern RC, Root AW. The reproductive system in cystic fibrosis. Basal gonadotropin and sex steroid levels. Am J Dis Child. 1981;135:422-6.

13. Reiter EO, Stern RC, Root AW. The reproductive system in cystic fibrosis. Changes in gonadotrophins and sex steroids following LHRH. Clin Endocrinol. 1982;16:127-37.

14. Aswani N, Taylor CJ, McGaw J, Pickering M, Rigby AS. Pubertal growth and development in cystic fibrosis: a retrospective review. Acta Paediatr. 2003;92:1029-32.

15. Cystic Fibrosis Foundation. Cystic Fibrosis Foundation Patient Registry: Annual Data Report 2009. Bethesda, Cystic Fibrosis Foundation; 2009.

16. Laursen EM, Juul A, Lanng S, Hoiby N, Koch C, Muller J, et al. Diminished concentrations of insulin-like growth factor I in cystic fibrosis. Arch Dis Child. 1995;72:494-7.

17. Staab D. Cystic fibrosis - therapeutic challenge in cystic fibrosis children. Eur J Endocrinol. 2004;151(Suppl 1):S77-80.

18. Boguszewski MC, Kamoi TO, Bento Radominski R, Boguszewski $\mathrm{CL}$, Rosberg S, Filho NA, et al. Insulin-like growth factor-1, leptin, body composition, and clinical status interactions in children with cystic fibrosis. Horm Res. 2007;67:250-6.

19. Colombo C, Battezzati A. Growth failure in cystic fibrosis: a true need for anabolic agents? J Pediatr. 2005;146:303-5.

20. Reiter EO. The KIGS experience with growth hormone treatment of cystic fibrosis. In: Ranke MB, Price DA, Reiter EO, editors.
Growth hormone therapy in Pediatrics -20 years of KIGS. Basel: Karger; 2007. p. 304-8.

21. Hardin DS, Adams-Huet B, Brown D, Chatfield B, Dyson M, Ferkol $T$, et al. Growth hormone treatment improves growth and clinical status in prepubertal children with cystic fibrosis: results of a multicenter randomized controlled trial. J Clin Endocrinol Metab. 2006;91:4925-9.

22. Hardin DS, Ellis KJ, Dyson M, Rice J, McConnell R, Seilheimer DK. Growth hormone decreases protein catabolism in children with cystic fibrosis. J Clin Endocrinol Metab. 2001;86:4424-8.

23. Hardin DS, Ellis KJ, Dyson M, Rice J, McConnell R, Seilheimer DK. Growth hormone improves clinical status in children with cystic fibrosis: results of a randomized controlled trial. J Pediatr. 2001;139:636-42.

24. Hutler M, Schnabel D, Staab D, Tacke A, Wahn U, Boning D, et al. Effect of growth hormone on exercise tolerance in children with cystic fibrosis. Med Sci Sports Exerc. 2002;34:567-72.

25. Schibler A, Heiden RVD, Birrer P, Mullis PE. Prospective randomized treatment with recombinant human growth hormone in cystic fibrosis. Arch Dis Child. 2003;88:1078-81.

26. Hardin DS, FerkolT, Ahn C, Dreimane D, Dyson M, Morse M, et al. A retrospective study of growth hormone use in adolescents with cystic fibrosis. Clin Endocrinol. 2005;62:560-6.

27. Hardin DS, Rice J, Ahn C, Ferkol T, Howenstine M, Spears S, et al. Growth hormone treatment enhances nutrition and growth in children with cystic fibrosis receiving enteral nutrition. J Pediatr. 2005;146:324-8.

28. Hardin DS, Ahn C, Prestidge C, Seilheimer DK, Ellis KJ. Growth hormone improves bone mineral content in children with cystic fibrosis. J Pediatr Endocrinol Metab. 2005;18:589-95.

29. Grasemann C, Ratjen F, Schnabel D, Reutershahn E, Vester U, Grasemann H. Effect of growth hormone therapy on nitric oxide formation in cystic fibrosis patients. Eur Respir J. 2008;31:815-21.

30. Schnabel D, Grasemann C, Staab D, Wollmann H, Ratjen F. A multicenter, randomized, double-blind, placebo-controlled trial to evaluate the metabolic and respiratory effects of growth hormone in children with cystic fibrosis. Pediatrics. 2007;119:e1230-38.

31. Darmaun D, Hayes V, Schaeffer D, Welch S, Mauras S. Metabolism in prepubertal children with cystic fibrosis: effects of glutamine and recombinant human growth hormone on protein. J Clin Endocrinol Metab. 2004;89:1146-52.

32. Aris RM, Merkel PA, Bachrach LK, Borowitz DS, Boyle MP, Elkin $\mathrm{SL}$, et al. Guide to bone health and disease in cystic fibrosis. J Clin Endocrinol Metab. 2005;90:1888-96.

33. Saggese G, Baroncelli Gl, Bertelloni S, Barsanti S. The effect of long-term growth hormone $(\mathrm{GH})$ treatment on bone mineral density in children with $\mathrm{GH}$ deficiency. Role of $\mathrm{GH}$ in attainment of peak bone mass. J Clin Endocrinol Metab. 1996;81:3077-83.

34. Phung OJ, Coleman Cl, Baker EL, Scholle JM, Girotto JE, Makanji SS, et al. Recombinant human growth hormone in the treatment of patients with cystic fibrosis. Pediatrics. 2010;126:e1211-26.

35. Wilson C. Cystic fibrosis-related diabetes. Nat Rev Endocrinol. 2011;7:375-8. 